\title{
UNUSUAL EVENT DETECTION AND LOCALIZATION USING OPTICAL FLOW TECHNIQUE
}

\author{
Taresh Singh $^{\# 1}$, Dr B M Singh ${ }^{* 2}$ \\ \# Dept. of Information Technology, College of Engineering Roorkee (Affiliated with UTU Dehradun) \\ Roorkee Uttarakhand (247667) \\ ${ }^{1}$ taresh.singhr@gmail.com \\ 2 dr.bmsingh1981@gmail.com
}

\begin{abstract}
Video-surveillance systems are becoming more and more independent in the finding and the exposure of anomalous events. The safety of mankind in crowds under disaster situation is one of the most essential concerns of researchers. This paper presents an approach to notice abnormal situations in crowded scenes by analysing the movement aspect and localize the unusual event using clusterization method . Experiments on UMN datasets show that the proposed method is promising.
\end{abstract}

Keyword - Unusual Events, Optical Flow, Context Specific, Gaussian Method.

\section{INTRODUCTION}

Video surveillance networks are growing rapidly due to the rising concern with the safety and security in public spaces. Main technological growth has been achieved in terms of using economical equipment, expert video stream management, and built-in processing and notification abilities in surveillance cameras. Developing a standard and scalable incident detection method for real-world scenario is a challenging problem due to unpredictability of scene type, viewing direction, nature of scene entities, and environmental conditions.

People in metropolitan areas have a higher probability of gathering together for sports, meetings, musical concerts, political elections rally etc. Under these situations, a lot of public gathering together in a comparatively small place, pedestrian density as a result become higher while pedestrian flow pattern becomes difficult. When pedestrian flow transfer from free flow to irregular flow and then to dangerous turbulent flow, which could induce very serious deaths and injuries. Situation might be even poorer under fire or natural hazards crisis. Taking fire evacuation in buildings as an instance, the movement of evacuees from different directions would mixed up in the lobby to the main exit, thus pedestrian flow might transfer from free flow to overcrowded flow, which would lower the efficiency of the total evacuation practice.

In this paper, we centred on avoiding context-specific information and propose a generic event modelling approach by mapping the event recognition problem to temporal data categorization problem. In this approach, classifier based event models are educated based on context invariant character to retain the detection ability in hidden scenarios without extra training and tuning. A fixed number of frame-level features are extracted indicating the global characteristics of the scene at a particular instant.

In the last years, most of supervision systems integrated computer vision algorithms to deal with problems like motion recognition and tracking. However, a few have treated the problems involving crowded scenes due to the challenging difficulty. Recently, the computer vision society has started taking concern in addressing different research problems related to the scenarios involving large crowds of people.

\section{RELATED WORKS}

In the last years, algorithms to deal with problems like motion recognition, tracking and unusual event detection have been developed.

Bruce et al. [1] explores image matching by the theory of differences. The method uses intensity differences between the images together with the spatial intensity gradient to contain from each image point linear constraints on the matching parameters. Barron et al. [2] reported the results of frequently cited techniques, including instances of differential, matching, energy-based, and phase-based approaches. They found that all techniques had problems at occlusion boundaries, not well reflected in the confidence measures. Beauchemin et al. [3] investigated the computation of widely known method for estimating optical flow are classified and examined by scrutinizing the hypotheses and assumptions they use. Hayashi et al. [4] used the Optical Flow Constraints (OFC) of edges to extract more precisely the flow of edges and to estimate the motion of the object using these flows. They found that the OFC approximates an edge by a step function, and therefore need to investigate its efficiency for other types of edge, such as a roof edge. Oliver et al. [5] proposed and compared two different state-based learning architectures, namely, HMMs and CHMMs for modelling behaviours and interactions. The CHMM model is shown to work much more efficiently and accurately. Stauffer et al. [6] have 
shown a novel, probabilistic technique for background subtraction. This technique deals with slow lighting changes by slowly adapting the values of the Gaussians and multimodal distributions caused by shadows, secularities, swaying branches, computer monitors, and other troublesome features of the real world which are not frequently mentioned in computer vision. Limitation of their method is that it works for selected areas and cannot classify the objects. Helbing et al. [7] used a model of pedestrian behaviour to investigate the mechanisms of panic and jamming by uncoordinated motion in crowds. Kamijo et al. [8] presents HMM-based event model was proposed for bumping, passing, jamming, illegal U-turn, and reckless driving detection using track features. Although the algorithm achieves such reliable tracking, it requires only gray-scale images. Lin et al. [9] developed a system goes one step further to estimate the number of people in crowded scenes in a complex background by using a single image. The detection tool can work well in some cases, it is still limited to some complex situations such as when the overlap among people is great and the contours of the heads are not clear. Rudolf Mester [10] shows how prior knowledge on the video signal can be integrated into a motion estimation procedure. Relations between different classes of motion algorithms are discussed and perspectives for a fusion and improvement of such procedures are presented. Niu et al. [12] proposed an algorithm for activity modelling and recognition from video clips captured at arbitrary views. They compared the use of only motion features, only shape features and both motion and shape features to build HMMs. It may not be capable to recognizing complex activities. Andrade et al. [13] applies a video modelling technique to a surveillance scenario where pedestrians are monitored to detect unusual events. It demonstrates that patterns derived from optical flow and encoded by a Hidden Markov Model are able to capture the dynamic evolution of normal behaviour allowing the classification of abnormal events. Pankaj Kumar et al. [14] present a rule-based framework for behaviour and activity detection in traffic videos obtained from stationary video cameras. These are classified into different categories using a novel Bayesian network approach, which makes use of image features and image-sequence-based tracking results for robust classification. It is capable of handling uncertainties that arise due to errors in visual signal processing. This system's performance can be further improved by having context images made more specific to the target. Armin Seyfried [15] presents the findings for the single-file movement with literature data for the movement in a plane. In the analysis presented they conclude the fundamental diagram for the single-file movement of pedestrians under laboratory conditions. The data are suitable to test, if microscopic models are able to reproduce the experiential relation between velocity and density in the simplest system. Andrade et al. [16] characterise crowd behaviour by observing the crowd optical flow and use unsupervised feature extraction to encode normal crowd behaviour. The experiments show that the bank of models is useful in quickly detecting the simulated emergency situation in a dense crowd. Andrade et al. [17] presented two different detection methods are implemented to detect global and local disaster scenarios. The hypothesis of having a dense crowd to detect enough flow changes can be lifted if the video sequences are modelled with banks of HMMs trained for normal motion in different density scenarios. This would be applicable only from modelling of dense crowds to pedestrian traffic. Blunsden et al. [18] presented an automatic technique for detection of abnormal events in crowds. Using projections of the eigenvectors in a sub-space spanned by the normal crowd scene as an input feature. The investigation of the relation between the number of eigenvectors and the model likelihood variations indicates that all configurations present a significant drop relative to the normal case and are able to correctly detect the emergencies. Masaaki et al. [19] describe a way for estimating optical flow from coloured images. In their approach, RGB data in the captured colour images are converted to the colour vectors. Since the colour vector has arbitrary orientation, the analysis of the optimal angle of the vector is reported in this paper. Min et al. [20] propose an algorithm that uses instantaneous motion field of the video instead of long-term motion tracks for learning the motion patterns. The motion field is a set of independent flow vectors identified in each frame of the video. Xiang et al. [21] developed a new visual behaviour modelling approach that performs incremental and adaptive model learning for online anomaly recognition in a visual surveillance scene. The approach has the following key features that make it useful over previous ones: (1) fully unsupervised learning. (2) Robust abnormality detection (3) Online and incremental model construction (4) Model adaptation to reflect changes in visual context. Antoni et al. [22] compared with state-of-the-art techniques in motion segmentation, together with temporal texture methods and conventional representations (e.g. optical flow or other localized motion representations), the combination of dynamic textures achieves superior performance in the problems of clustering and segmenting video of such processes. Nacim et al. [23] Identified events where local motion variation is significant compared to earlier events. Optical flow methods are used to extract information such as compactness, direction and velocity. It is likely to extend the estimation of the motion variations with factors such as acceleration by tracking the Points Of Interest (POIs) over various frames. Amit et al. [24] proposed a new algorithm for recognition of certain types of abnormal events. In this algorithm multiple local monitors to collect low-level statistics and produces an alert these alerts are incorporated to an ultimate decision regarding the existence of an unusual event. The main limitation of their algorithm is the lack of sequential monitoring. Ramin et al. [26] proposed a technique to identify and localize unusual behaviours in crowd videos using Social Force model. A grid of particles is placed over the image and it is advected with the space-time average of optical flow. They categorize frames as usual 
and unusual by using a bag of words technique. The regions of anomalies in the unusual frames are localized using communication forces. Tian et al. [27] proposed a technique for the detection of anomaly in busy scenes based on the crowd movement description. These description include the crowd kinetic energy and the movement directions. This estimates the crowd kinetic energy and the motion directions using the optical flow techniques. Wang et al. [28] presented for abnormal sections recognition in video sequences using the histogram to illustrate the colour alteration in the segment, and the histograms of the frames identified from the section create the histogram matrix. To improve the procedure effectiveness, the principal components analysis (PCA) is used to decrease size of the histogram matrix. The information of frames differences is used to illustrate the motion variation information, and the information twice frame differentiation is used to illustrate the rate of change. WALI et al. [29] proposed a new method for event recognition from video surveillance information using optical flow histogram without previous information of the motion pattern. They begin by estimating the motion from images series by optical flow technique. Second, followed by classification using the histogram of the optical flow vectors and using a chain coding algorithm. At last, they extract a high-level feature from any frame for use in the learning and investigate events by SVM and HMM. Sukeshini et al. [30] presented optical flow motion vector estimation using iterative Lucas-Kanade Pyramidal implementation for both large \& small motion. The velocity \& displacement at every pixel is obtained by using Lucas-Kanade equations. The original image is recovered by warping reference frame towards current frame by flow vectors i.e. velocity \& displacement by using image warping techniques. Shu Wang et al. [31] proposed a method to automatically identify anomalous behaviour in crowd scene. They separated whole frame into small blocks, and movement pattern in each chunk is encoded by the distribution of motion vectors in it. Similar motion patterns are grouped into pattern model in an unsupervised way, and they categorize motion pattern into usual or unusual group according to the variation between motion pattern and trained model. It is required to make the descriptor stronger in poor lighting scene. Julio et al. [32] presented a study on crowd analysis based on computer vision. This study focused on three important problems in crowd analysis: people counting/density estimation, tracking in crowded scenes, and crowd behaviour understanding in a higher-level analysis, like the temporal evolution, main directions, velocity estimations, and recognition of abnormal situations. Abhijit et al. [33] proposed a real time moving entity recognition algorithm from a single freely moving monocular camera. An online visual SLAM algorithm working at the same time estimates the camera egomotion. Christoph et al. [34] used a twostage mean-shift algorithm for detection of all moving objects in the scene by an image-based mean-shift which utilises a grey value histogram as a target model and refinement of the object pose largely along the depth axis. The prediction step in the tracking system relies on flow-based, kinematic, and trajectory-based prediction methods. Cen Rao et al. [35] present a view-invariant illustration of movement having dynamic instants and intervals that is computed using spatiotemporal curvature of a path. Then this illustration is used by their method to learn human behaviour without any training. The system automatically segments video into individual behaviour, and computes view invariant illustration for each action. Lili Cui et al. [37] presented an anomalous event recognition technique using local features for traffic video surveillance. Each forefront region's area, shape factors and pixel affecting velocity vector are extracted. Using these character, regions are classified into dissimilar groups as pedestrian, vehicle or noise region, and their actions is classified using trained local features' distribution map. Finally, a simple classifier is used to decide objects' states of usual or unusual. Su Yang et al. [38] presented a case study of detecting unusual traffic flows and find out new resolution by presentation the coevolving data streams as high-dimensional patterns and applying various learning techniques to capture the description of such patterns. Xiaobai Liu et al. [39] proposed a flexible structure that combines the spatiotemporal contextual knowledge with a novel deformable pattern matching process. In this process, the background modelling was scene-specific and thus not flexible enough for portable visual surveillance system. Duan-Yu Chen et al. [40] estimated optical flows and used to find out cluster of human crowds in unconfirmed manner using their proposed scheme of adjacency-matrix based clustering (AMC). Based on clusters of human crows, their characters with properties, direction, location and crowd size, are considered by a model of force field. Finally, they predicted the characters of human crowds and then detect if any abnormalities of human crowd there in the scene. Mahfuzul et al. [41] proposed a common event recognition method in which the extracted low-level characters signify the global features of the target scene instead of any context-specific information. In this method, supervised training and thorough feature extraction are necessary only once while construction the target event models. Hajananth et al. [42] presented a model using a Semi-2D Hidden Markov Model (HMM) to the usual actions of people. Their Semi-2D HMM is intended to model both the temporal and spatial causalities of the crowd activities by assuming the present state of the Hidden Markov Model depends not only on the preceding state in the of time direction, but also on the earlier states of the nearby spatial locations. Tian Wang et al. [43] proposed an algorithm to identify unusual events based on histograms of the orientation of optical flow illustrator and one-class SVM classifier. They presented grids of Histograms of the Orientation of Optical Flow (HOFs) as the descriptors for movement information of the colossal video frame. 
Hiral Raveshiya et al. [44] presented summary of some fundamental concepts related to motion estimation using optical flow estimation processes and activity estimation techniques are attractive research area which is described here in brief. Sharmin et al. [45] presented an image filtering scheme as a pre-processing stage for the Lucas-Kanade pyramidal optical flow algorithm. Based on a study of dissimilar types of filtering techniques and applied on the Iterative Refined Lucas-Kanade, resulted on the best filtering exercise. As the Gaussian smoothing filter was selected, an experiential approach for the Gaussian variance estimation was introduced.

Oluwatoyin et al. [46] focuses on relative unusual human behaviour recognition in particular in video surveillance applications. The main reason of this review is to widely recognize existing techniques and distinguish the literature in a method that brings key challenges to notice. Hajananth et al. [47] studied the performance of different state of the art features to identify the occurrence of the unusual objects in the scene. These comprise optical flow vectors to detect motion related abnormalities, textures of optical flow and image textures to identify the occurrence of anomalous objects. Hiba et al. [48] presented a novel automated algorithm for the Detection of Abnormal activities in Dynamic Crowded Gatherings (DADCG) that has diminished processing speed, understanding to noise, and enhanced precision. Firstly, the temporal features of the scenes are extracted using Motion History Image (MHI) technique. Then the Optical Flow (OF) vectors are calculated for each MHI image using Lucas-Kanade method to get the spatial character. This Optical flow image is segmented into four equal-sized segments. Finally, a two dimensional histogram is calculated with motion track and motion scale for each chunk. Weiguo et al. [49] studied the scheme and technology for executing and analyzing two critical controlled experiments. Based on the study and some typical characteristics of the singlefile experiment, a one dimensional continuous distance model (CDM) was projected in their earlier work and in this study it is improved by introducing the needed moving direction so as to describe the two-dimensional bottleneck movement. Nguyen et al. [50] presented a method that include Brox's optical flow algorithm with background subtraction with a new method to get better optical flow in the traffic surveillance system.

Malavika et al. [51] presented an implementation of image subtraction algorithm to identify a moving object and the velocity has been calculated. Dhara et al. [52] presented general idea of some elementary concepts associated to motion estimation and presented the Lucas kanade method which is broadly used in differential method for optical flow estimation and computer vision. Soheil et al. [53] proposed a comparative study of Cepstrum, principal component analysis (PCA) and symbolic dynamic filtering (SDF) as feature extractors for target detection and classification. These methods have been tested on two data sets, consisting of passive infrared (PIR) and seismic sensors, collected from different fields. The underlying algorithms of feature extraction have been executed in conjunction with three different classification algorithms, namely, support vector machines (SVM), k-nearest neighbour (k-NN), and sparse representation classifier (SRC). Crossvalidation has been used to assess the performance of Cepstrum, PCA and SDF as feature extractors for both PIR and seismic sensor data sets. Jorge et al. [54] presented a scheme for unusual activity investigation that ensures efficiency in the inferences with less computational effort. They use a region-based model that uses a mobile grid of sub regions constructed from scene's ROI (region of interest). Rajanayaki et al [55] presented model to identify the global actions in crowd through observing motion patterns such as flow, velocity and direction. Background subtraction as a pre processing step is performed to extract the foreground blobs and optical flow is calculated to find the velocity and direction of motion. The human crowds are then clustered based on alike direction and nearness using Adjacency Matrix based Clustering (AMC). Chunyu et al. [56] concentrated on the challenges of recognition and localization of crowd escape abnormal behaviours in video surveillance process. This scheme identifies the unusual events as well as identifies the potential location of unusual events. Tian et al. [57] introduced a histogram of optical flow orientation as a descriptor encoding and nonlinear one-class support vector machine classification algorithm, subsequent a learning period characterizing the usual behaviour of training frames, recognizes unusual events in the present frame. Aravinda et al. [58] presented crowd activity recognition and classification in optical flow methods (OFMs) to identify walking and running events, which uses optical flow vector lengths in OFMs. Prabuwono et al. [59] presented a two-layer bucket method using moment-based feature extraction algorithm (MFEA). A moment represents the coherent intensity of pixels and motion estimation is a motion pixel intensity measurement, this research used this relation to develop the proposed algorithm.Their approach contributes to the detection of abnormal events in crowd flows by the fact that it doesn't need learning process and training data, it also considers simultaneously density, direction and velocity and focuses analysis on specific regions where the density of motions is high. Weixin et al. [60] proposed a detector based on a video representation that accounts for both appearance and dynamics, using a set of mixture of dynamic textures models. A data set of densely crowded pedestrian walkways is introduced and used to evaluate the proposed anomaly detector. 


\section{III.PROPOSED APPROACH}

In this section, different processing components of the proposed event detection framework are presented.

\section{A. Lucas-Kanade Method for Optical Flow Measurement}

The Lucas-Kanade method is broadly used in differential method for optical flow estimation and computer vision. This method solves the fundamental optical flow equations for all the pixels in that neighbourhood, by the least squares criterion. It is a purely local process because it cannot offer flow knowledge in the interior of consistent regions of the image. Here assumes that the flow is essentially constant in a local neighbourhood of the pixel under consideration. The Lucas-Kanade process assumes that the displacement of the image contents between two consecutive frames is small and approximately constant within a neighbourhood of the point $p$.

$$
\begin{gathered}
I_{x}\left(p_{1}\right) V_{x}+I_{y}\left(p_{1}\right) V_{y}=-I_{t}\left(p_{1}\right) \\
I_{x}\left(p_{2}\right) V_{x}+I_{y}\left(p_{2}\right) V_{y}=-I_{t}\left(p_{2}\right) \\
\cdot \\
\cdot \\
I_{x}\left(p_{n}\right) V_{x}+I_{y}\left(p_{n}\right) V_{y}=-I_{t}\left(p_{n}\right)
\end{gathered}
$$

Where $\mathrm{p}_{1}, \mathrm{p}_{2} \ldots \mathrm{p}_{\mathrm{n}}$ are pixels inside the window, $I_{x}\left(p_{n}\right), I_{y}\left(p_{n}\right), I_{t}\left(p_{n}\right)$ are the partial derivatives of the image $I$ with respect to position $x, y$ and time $t$, computed at the point $p_{n}$ and at the current time. The equations can be written in matrix form:

$$
\begin{array}{cr}
\mathrm{Av}=\mathrm{b} & \text { Where } \\
\mathrm{A}=\left[\begin{array}{c}
I_{x}(p 1) I_{y}(p 1) \\
I_{x}(p 2) I_{y}(p 2) \\
\vdots \\
I_{x}(p n) I_{y}(p n)
\end{array}\right] \mathrm{v}=\left[\begin{array}{c}
V_{x} \\
V_{y}
\end{array}\right] \mathrm{b}=\left[\begin{array}{c}
-I_{t}(p 1) \\
-I_{t}(p 2) \\
\vdots \\
-I_{t}(p n)
\end{array}\right]
\end{array}
$$

Based on the least squares principle to find out the solution of Lucas-kanade method. Now $A^{T} A V=A^{T} b$, this equation can also be written in this form, so we get:

$$
\mathrm{V}=\left(\mathrm{A}^{\mathrm{T}} \mathrm{A}\right)^{-1} \mathrm{~A}^{\mathrm{T}} \mathrm{b}
$$

Where $A^{T}$ is the transpose of matrix $\mathrm{A}$, so we can get,

Where $n=1$ to $N$

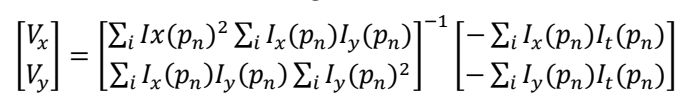

The matrix $A^{T}$ is often called the structure tensor of the image at the point $p$. Weighted window: The plain least squares solution above gives the similar importance to all $\mathrm{N}$ pixels $p_{n}$ in the window. Practically it is generally better to assign more weight to the pixels that are nearer to the central pixel $p$. For that, one uses the weighted version of the least squares equation.

$$
A^{T} W A V=A^{T} W b
$$

We can also write this equation in this form, so we get:

$$
V=\left(A^{T} W A V\right)^{-1} A^{T} W b
$$

Where $W$ is an $n x n$ diagonal matrix having the weight $W i i=w i$ to be given to the equation of pixel $p_{n \text {. }}$ It computes:

$$
\left[\begin{array}{l}
V_{x} \\
V_{y}
\end{array}\right]=\left[\begin{array}{l}
\sum_{i} I x\left(p_{n}\right)^{2} \sum_{i} I_{x}\left(p_{n}\right) I_{y}\left(p_{n}\right) \\
\sum_{i} I_{x}\left(p_{n}\right) I_{y}\left(p_{n}\right) \sum_{i} I_{y}\left(p_{n}\right)^{2}
\end{array}\right]^{-1}\left[\begin{array}{l}
-\sum_{i} I_{x}\left(p_{n}\right) I_{t}\left(p_{n}\right) \\
-\sum_{i} I_{y}\left(p_{n}\right) I_{t}\left(p_{n}\right)
\end{array}\right]
$$

The weight $w_{i}$ is generally set to a Gaussian function of the distance between $p_{n}$ and $p$. Lucas-Kanade and others implemented a weighted least squares (LS) fit of local first-order constraints to a constant model for $v$ in every small spatial neighbourhood by minimizing

$$
\sum w^{2}(x, y)\left[\nabla I(x, y, t) v+I t(x, y, t]^{2}\right.
$$

Where $\mathrm{w}(\mathrm{x}, \mathrm{y})$ indicates a window function that gives additional influence to constraints at the centre of the neighbourhood.

B. Energy of Motion Detection Algorithm (EMDA)

1. Video Stream input

2. Repeat step 1 to 7

3. Convert RGB frame to Gray Scale 
4. Estimate optical flow

5. Find the energy magnitude

6. If (magnitude>threshold)

Abnormal Event Detected

7. Else Normal Activity

\section{Abnormality Detection Based on the Energy of the Optical Flow}

Abnormal behaviours generally have features such as big motion magnitudes, fast velocities, and various directions and so on. While the motion magnitudes of the usual behaviours are not big and the motion directions are roughly same, consequential to the motion energy is low in one direction. In the case of anomalous behaviours, due to the litter of motion direction and large speed in the process of movement, the kinetic energy of speed must be higher than the usual behaviours. Thus the kinetic energy of optical flow is useful to reaction the behavioural characteristic. In this paper, the kinetic energy of $n^{\text {th }}$ frame optical flow is extracted using the following equation:

$$
E(n)=\sum_{i=1}^{W} \sum_{j=1}^{H} W_{i, j}(n) V_{i, j}^{2}(n)
$$

$E(n)$ depicts the kinetic energy of the optical flow in the movement region. Parameter $V_{i, j(n)}$ is the velocity of the $n^{\text {th }}$ frame image pixels pix $(i, j)$ and coefficient $w_{i, j}(n)$ is the weight of the optical flow energy. $W$ denotes the width of the movement area and $H$ denotes the height of the movement area. The velocity of each pixel in the movement area is calculated using following equation:

$$
v_{i, j}(n)=\sqrt{\left|\Delta X_{i, j}\right|\left|\Delta Y_{i, j}\right|}
$$

The offsets of horizontal direction and vertical direction are $\Delta X_{i, j}$ and $\Delta Y_{i, j}$ respectively. The angle between the moving direction and the horizontal direction is denoted as:

$$
\operatorname{Angel}_{i, j}(n)=\arctan \frac{\Delta Y_{i, j}}{\Delta X_{i, j}}
$$

The optical flow includes instantaneous speed as well as movement direction of each pixel. The magnitude of velocity shows the intensity of movement, and the motion direction angle can used to find the direction of moving target. In the case of the usual behaviour, the motion directions are generally same and the difference between pixels is not large. On the contrary, the directions of movement are not in order and not steady, the movement with a high and strong magnitudes for the unusual behaviours. Related with the characteristics of the optical flow, this point outs the variation of pixels speeds and the variation of the directions of motion. Therefore, for the non-weighted optical flow, it does not differentiate the unusual behaviours and the usual behaviours properly only by using the knowledge of the optical flow speed. As per the above discussion, we describe the weight of the optical flow energy:

$$
\begin{gathered}
\mathrm{w}_{\mathrm{i}, \mathrm{j}}(\mathrm{n})=\left(\frac{\Delta \text { Angel }}{\pi} \times \alpha\right)^{2}+\left(\frac{\Delta \text { AngelMax }}{\pi} \times \alpha\right)^{2} \\
\Delta \text { Angel }=\mid \angle \text { AngelCurrent }-\angle \text { AngelAvg } \mid \\
\Delta \text { AngelMax }=\mid \angle \text { AngelCurrent }-\angle \text { AngelMax } \mid
\end{gathered}
$$

Where $\angle$ AngelCurrent indicates the angel between the present pixel and the horizontal direction. The angle of the max speed pixel is $\angle$ AngelMaxin the movement region. The average angle between moving target and horizontal direction:

$$
\angle \text { AngelAvg }=\frac{\sum \text { Angel }_{i, j}}{\operatorname{Sum}(\mathrm{P})}
$$

Where Sum $(P)$ indicates the sum of all pixels in the movement region.

\section{IV.EXPERIMENTAL RESULTS}

In this section, we test our algorithm on the real data is the public dataset: the UMN dataset which have been widely used for performance evaluation. Most of the parameters associated with the proposed method are set to fixed values. The video frame size is $240 \times 320$ pixels, data rate $4500 \mathrm{kbps}$ and frame rate $30 \mathrm{frame} / \mathrm{second}$. In our experiment, we tested our algorithm on Intel Pentium $2.66 \mathrm{GHz}$ quad core processor with 2 GB RAM.

We show the results based on the energy of motion using optical flow estimation on the UMN dataset. We compare results with the Horn-Schunck Method for determining the unusual events. We estimate optical flow using Lucas-Kanade derivative of Gaussian method. First we have converted the RGB video in to gray scale video so that it occupies low space on disk. Due to this conversion the processing cost of the video become very low and system will be very efficient. 


\section{A. Energy Detection on the UMN Dataset}

The UMN dataset are shot in two different scenes, including both indoor and outdoor. The dataset contains 11 different scenarios of an escape event. Each video clip first depicts a crowd of people walk around or stay in a lobby, and then move away from the field of view. Fig. 1(a), 1(b), 3(a) and fig. 3(b) shows some sample frames and fig. 2 the results of detection. We display the results of two different scenes and the corresponding frames which the escape events happened of each scene. From Fig. 2 and fig. 5, we can find a wave crest clearly. In The energy curve of the optical flow begins to increase at about 170th frame in Figure 2 and $270^{\text {th }}$ in Figure 4 when comparing to the video data, the crowd of people start to escape. The energy curve reaches its peak at about 180 th frame in figure- 2 and $300^{\text {th }}$ frame in figure-4. After 180th frame in figure- 2 and $300^{\text {th }}$ frame in figure- 4 , with the people leaving out the scene, the curve begins to decrease.

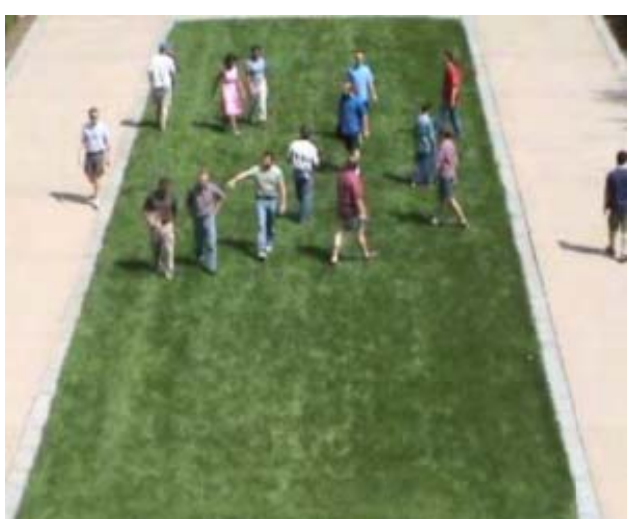

Fig-1(a) usual event

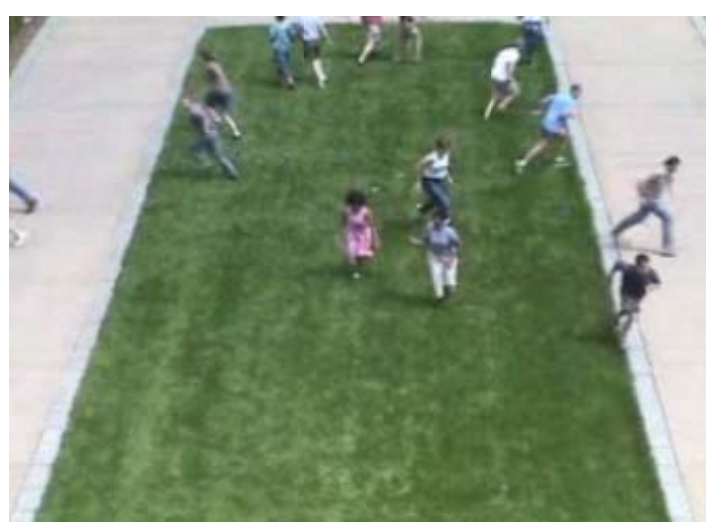

Fig-1(b) unusual event

Scene at lawn

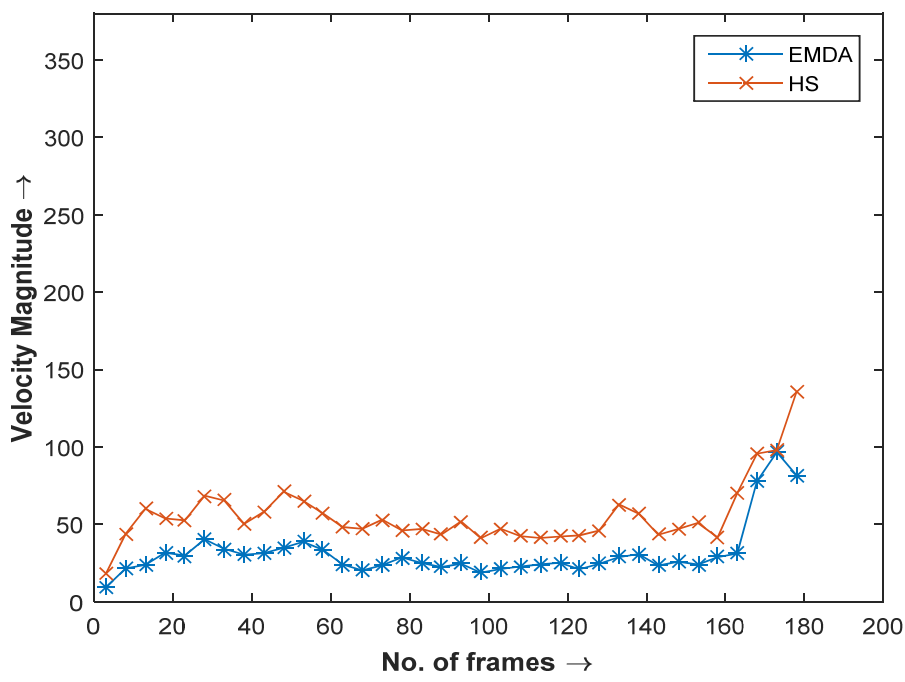

Fig-2 Comparative result of unusual event detection

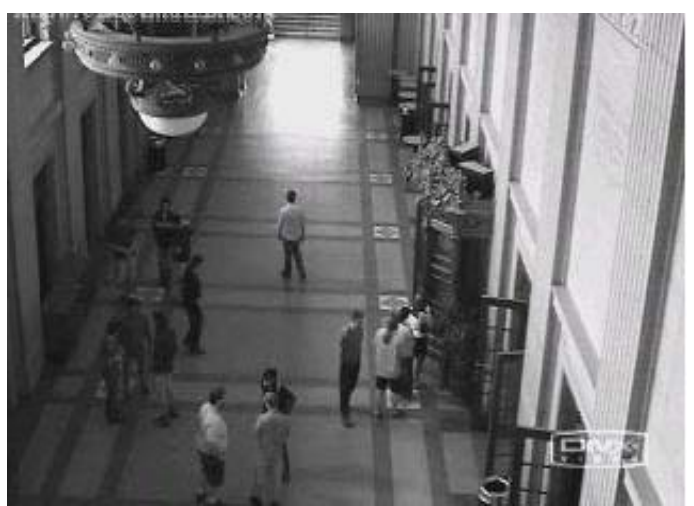

Fig-3(a) usual event

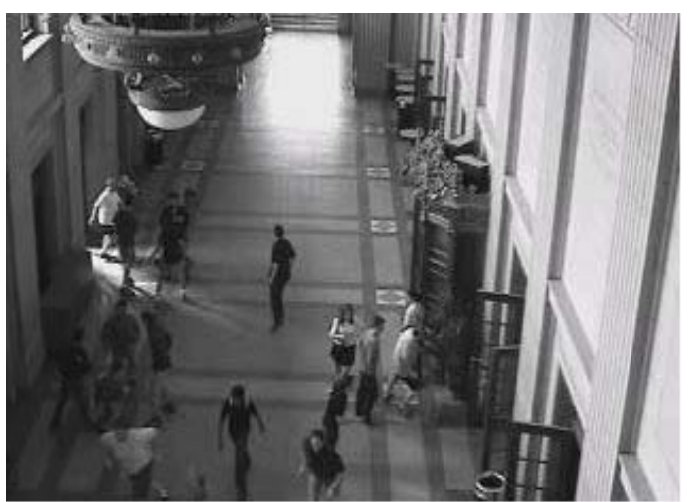

Fig-3(b) unusual event 
Scene at corridor with variable illumination

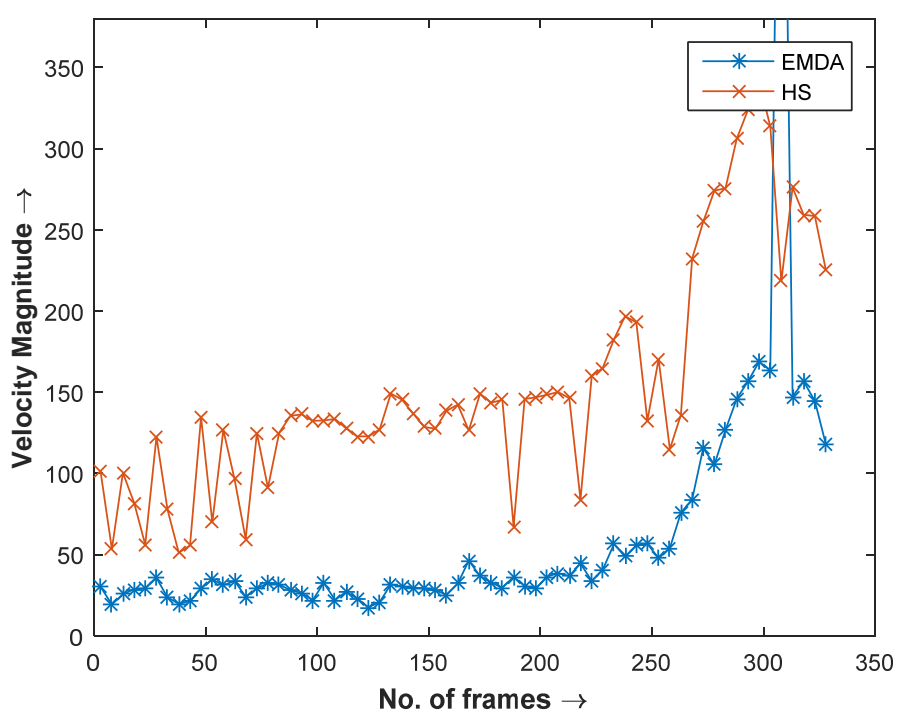

Fig-4 Comparative result of unusual event detection at variable illumination

From Figure 2 and fig. 4, the proposed approach successfully detected the trend of the abnormality. However, in order to get an accurate output, it is necessary to choose the suitable threshold.

\section{B. Unusual Event Localization:}

A few researches have been studied for the localization of anomalous crowd behaviours in videos. Some literatures about the localization are almost only study the individual anomalies. However, the consequence caused by the anomalous crowd behaviours is more severe, so determining the location of the anomalous crowd behaviours is very important to take action timely when the unusual events happen. For the abnormal crowd events localization, literature has been mentioned that the Markov Chain Monte Carlo method is used to address the localization issue. We consider the problem of abnormal crowd events localization, the notion of the divergent centre is inspired by the idea of literature [60]. We proposed a clusterazation method to determine the divergent centre.

We test our approach on public database and some realistic video recorded by ourselves: public database including the UMN database. UMN database includes 3 videos in 3 different scenes: lawn, indoor and square. All these 3 videos depict people wandering in the beginning and suddenly evacuate quickly at a time. Every video of UMN database video has a relative long time of normal situation in the beginning and short abnormal situation in the end.

In the proposed method first starting frame of unusual event is detected using optical flow technique. Then optical flow vector is divided into small clusters. On the bases of velocity magnitude of each cluster it can detect the location of unusual event.

As shown in fig 5(a), fig 6(a), and fig 7(a) the snap shot of usual event and optical flow of the frame and fig 5(b), fig 6(b) and fig 7(b) snap shot of unusual event optical flow of the frame. Finally in fig 5(c), fig 6(c), and fig 7(c) represent the surface plot of the unusual event. In table-1, table-2, table-3 the cluster wise velocity magnitude tables are shown correspondingly. 

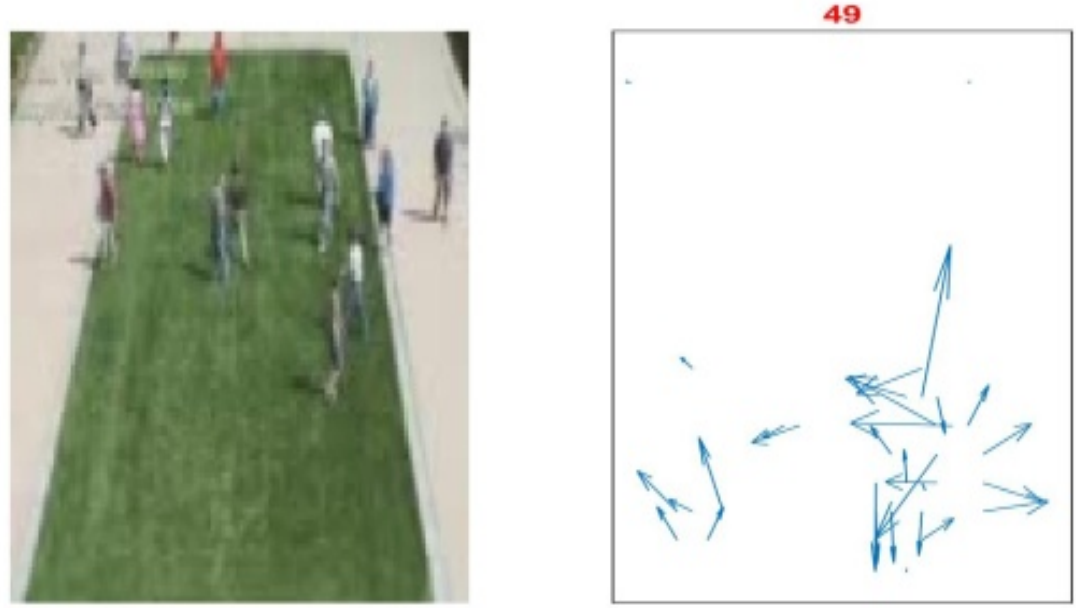

Fig 5(a) Usual Event

167Unusual Event
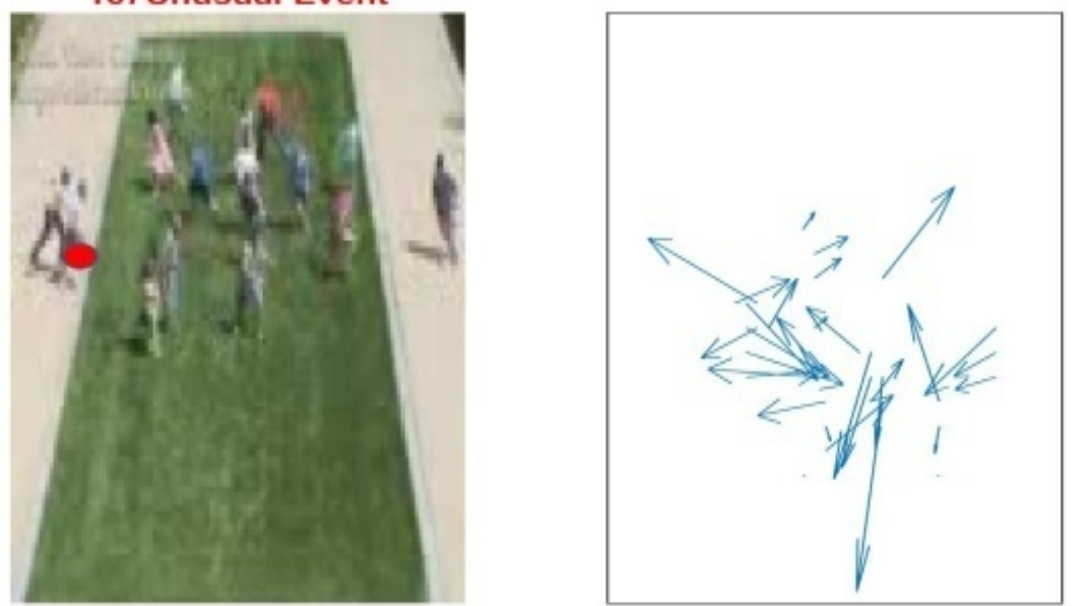

Fig 5(b): Unusual Event

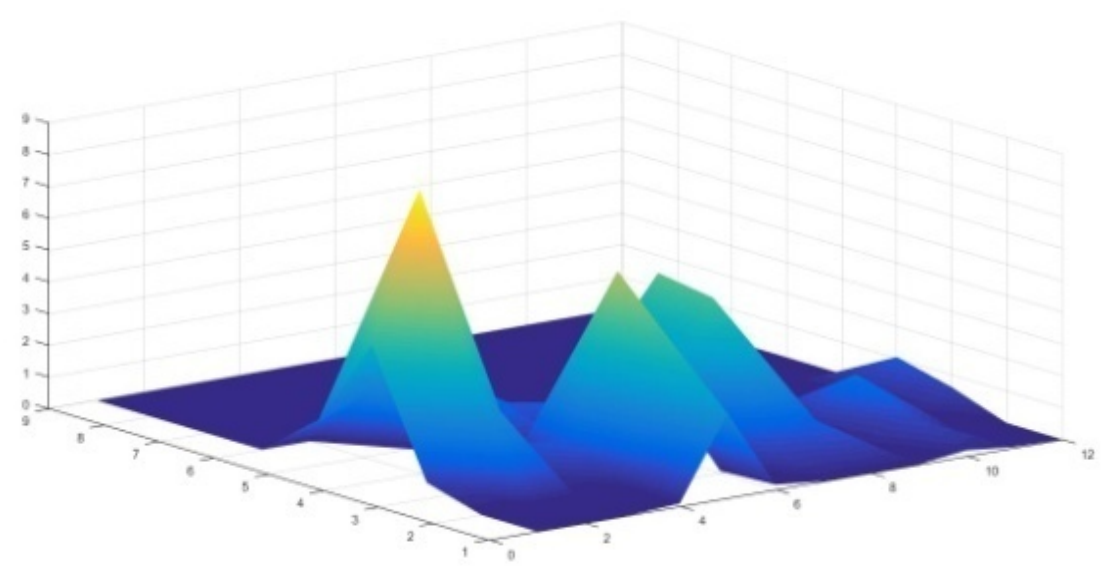

Fig5 (c): Surface Plot of Unusual Event 

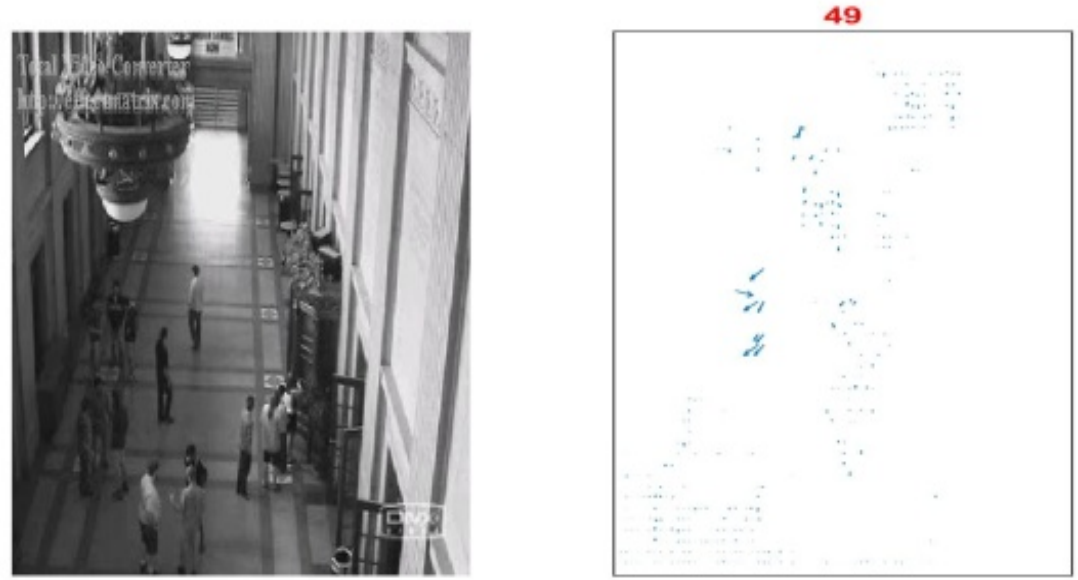

Fig 6: Usual Event
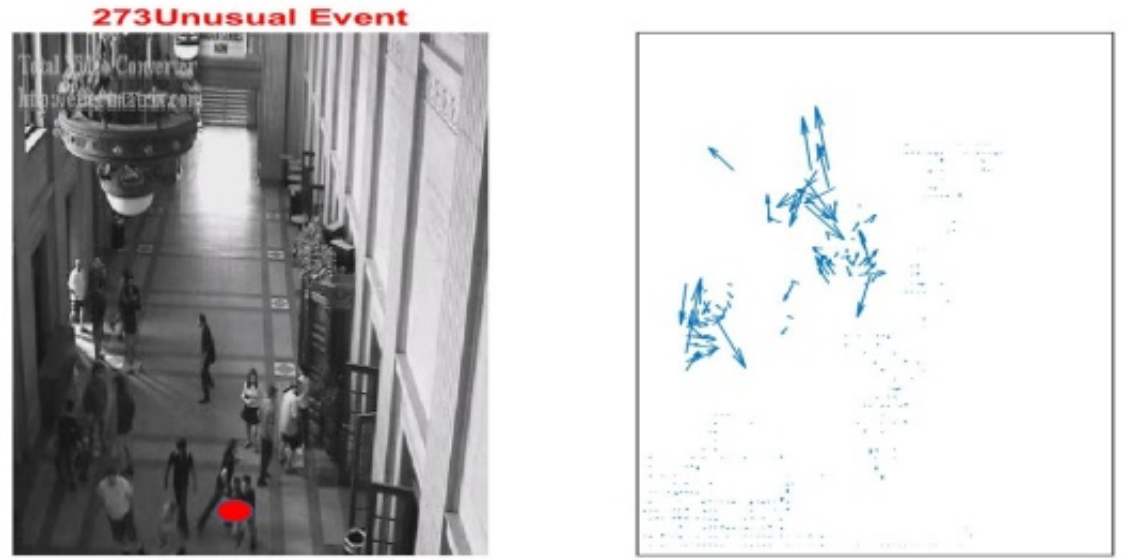

Fig 6(b): Unusual Event

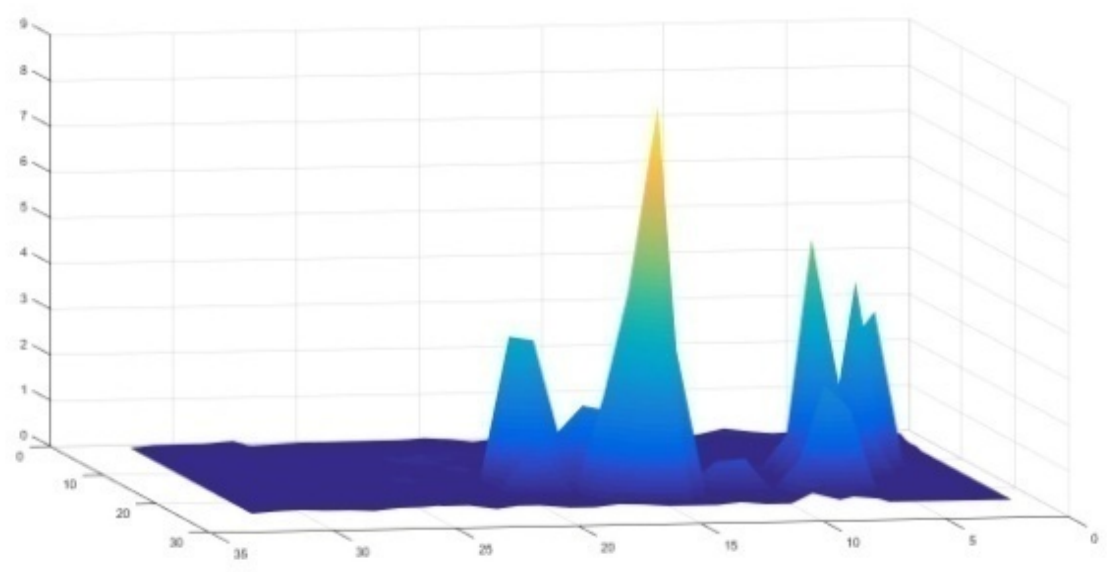

Fig 6(c): Surface Plot of Unusual Event 

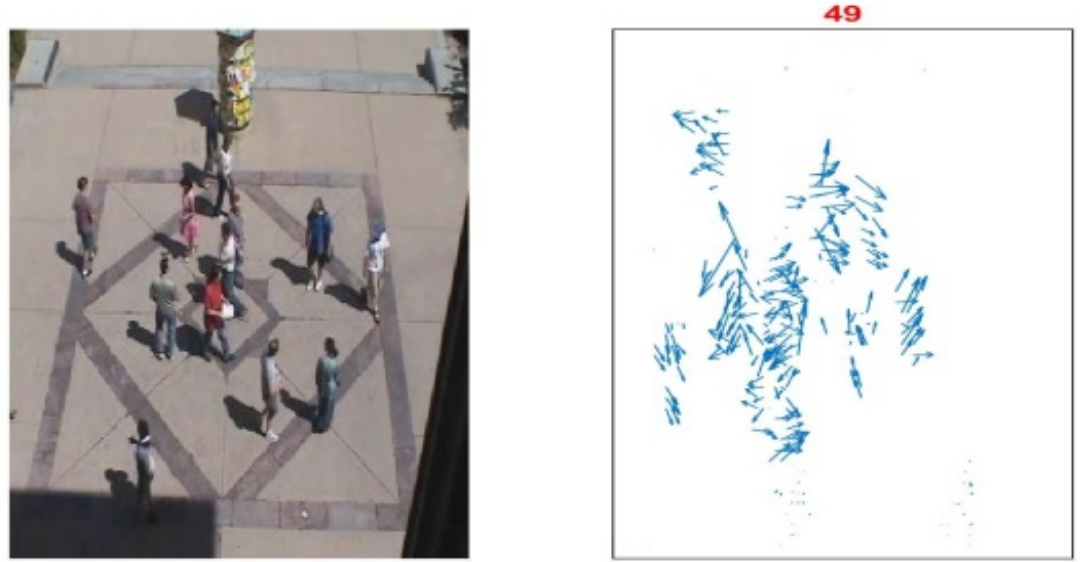

Fig 7(a): Usual Event
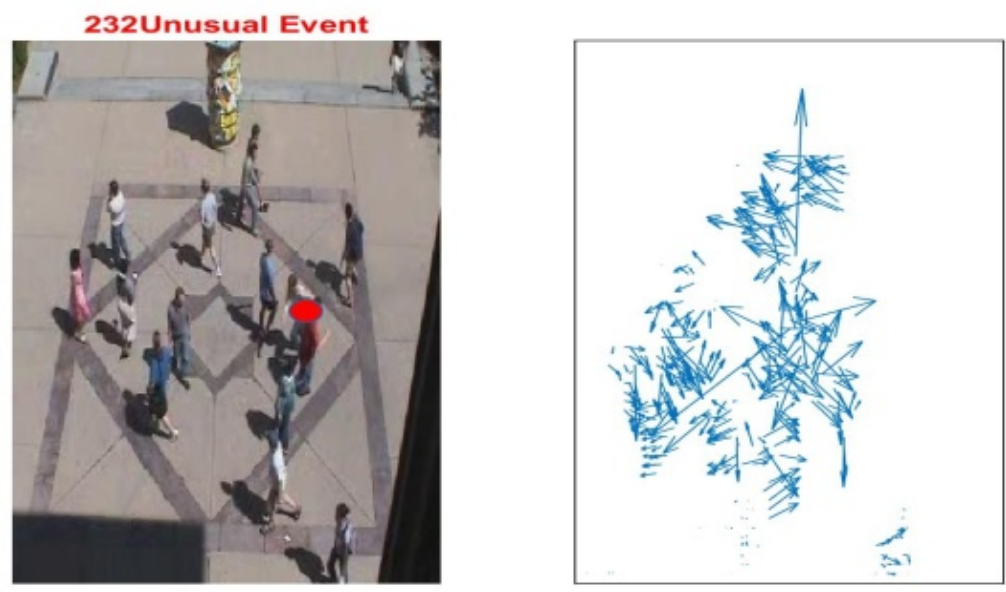

Fig 7(b): Unusual Event

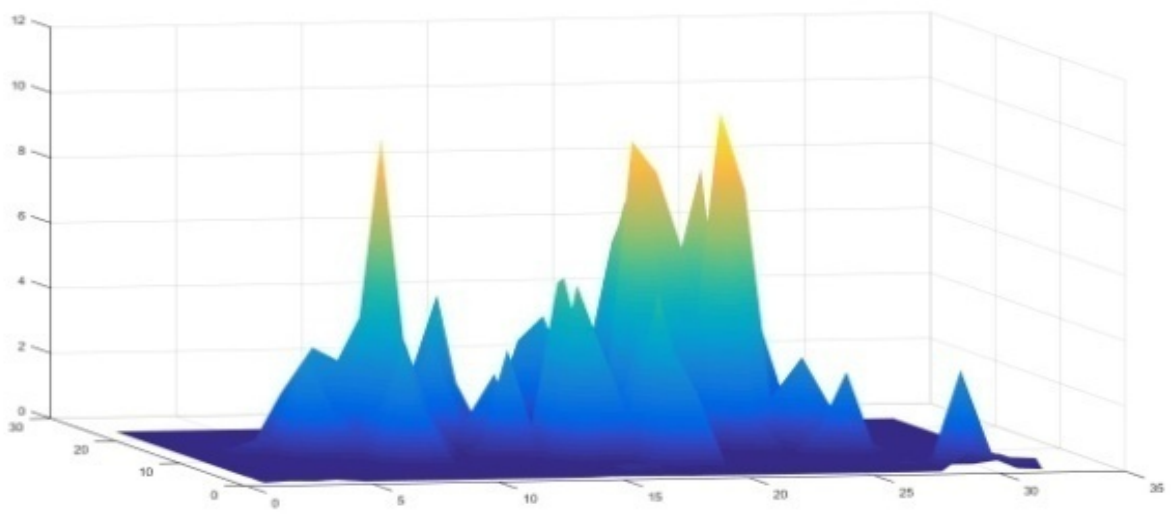

Fig 7(c): Surface Plot of Unusual Event 
Table-1: Cluster-wise Magnitude of Velocity Corresponding to fig-5

\begin{tabular}{|r|r|r|r|r|r|r|r|r|r|r|r|r|r|r|}
\hline & 1 & 2 & 3 & 4 & 5 & 6 & 7 & 8 & 9 & 10 & 11 & 12 \\
\hline 1 & 0 & 0 & 0.0030537 & 0.0868164 & 2.661453 & 0.155793 & 0 & 0 & 0 & 0.1797456 & 0.0257375 & 0 \\
\hline 2 & 0 & 0 & 0 & 0 & 4.351769 & 0.068607 & 0.848035 & 1140515 & 0 & 0.7011887 & 0 & 0 \\
\hline 3 & 0.5065873 & 3.420501 & 0.358693 & 1.0536406 & 6.122131 & 2.080917 & 4.749203 & 0.418344 & 0.1650282 & 154417 & 0 & 0.614899 \\
\hline 4 & 4.3347716 & 8.955802 & 0.5855963 & 0 & 0.283273 & 1183335 & 5.017273 & 0.953796 & 0 & 0.1330498 & 0 & 1.048037 \\
\hline 5 & 13308564 & 1971362 & 0 & 1897254 & 0.95191 & 0 & 0.423434 & 0.366005 & 0.3555289 & 0 & 0 & 0 \\
\hline 6 & 0 & 0 & 0 & 0.8386505 & 0.281352 & 0 & 0 & 0 & 0 & 0 & 0 & 0 \\
\hline 7 & 0 & 0 & 0 & 0 & 0 & 0 & 0 & 0 & 0 & 0 & 0 & 0 \\
\hline 8 & 0 & 0 & 0 & 0 & 0 & 0 & 0 & 0 & 0 & 0 & 0 & 0 \\
\hline 9 & 0 & 0 & 0 & 0 & 0 & 0 & 0 & 0 & 0 & 0 & 0 & 0 \\
\hline
\end{tabular}

Table-2: Cluster-wise Magnitude of Velocity Corresponding to fig-6

\begin{tabular}{|c|c|c|c|c|c|c|c|c|c|c|c|c|c|c|c|c|c|c|c|c|c|c|c|c|c|c|c|c|c|c|c|}
\hline & & & & & & & & & & 10 & ${ }_{11}$ & 12 & 13 & 14 & 15 & 16 & 17 & 18 & 19 & 20 & 21 & 22 & 23 & 24 & 25 & 26 & 27 & 28 & 29 & 30 & $31=$ \\
\hline & 0.0363 & \begin{tabular}{|l|l|}
0.0436 \\
\end{tabular} & \begin{tabular}{|l} 
\\
\end{tabular} & 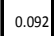 & 0.0823 & \begin{tabular}{|l|} 
\\
\end{tabular} & 0.0085 & 0.0237 & 0.044 & 0.0825 & 0.112 & 0.071 & 0.086 & 0.038 & 0.068 & 0.178 & 0.057 & & 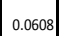 & 0.0978 & 0.063 & 0.061 & 0.054 & 0.049 & 0.05288 & \begin{tabular}{|l}
0.0265 \\
\end{tabular} & 0.00157 & 0.0645 & \begin{tabular}{|l|l|}
0.0627 \\
\end{tabular} & 0.06 & 0.051 \\
\hline & 0.0368 & \begin{tabular}{|l|l|} 
& 0.0433 \\
\end{tabular} & \begin{tabular}{|l|l|} 
& 0.1517 \\
\end{tabular} & $\mid 0.245$ & $\begin{array}{l}0.1162 \\
0\end{array}$ & 0.118 & 0.18 & 0.2522 & 0.115 。 & 0.1155 & 0.138 & 0.12 & 0.121 & 0.008 & 0.039 & 0.03 & 0.007 & & & 0 & 0 & & & & & 0.02525 & & 0.1785 & $\begin{array}{l}0.0073 \\
\end{array}$ & & 0.066 \\
\hline & B 0.1369 & \begin{tabular}{|l|l|} 
\\
\end{tabular} .0818 & \begin{tabular}{|l|l|} 
\\
\end{tabular} & \begin{tabular}{|l|l|} 
\\
\end{tabular} & 0.0747 & 0.177 & 0.18 & 0.0285 & 0.035 。 & 0.0739 & 0.067 & 0.086 & 0.129 & & & 0 & & & & & 0.014 & & & & & & & & & & \\
\hline & \begin{tabular}{l|l}
4 & 0.0558
\end{tabular} & \begin{tabular}{|l|l|} 
& 0.1144 \\
\end{tabular} & \begin{tabular}{|l} 
\\
0.1619
\end{tabular} & \begin{tabular}{|l|l|} 
& 0.197 \\
\end{tabular} & 0.1931 & \begin{tabular}{|l|l|} 
& 0.129 \\
\end{tabular} & 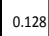 & 0.0346 & 0.1490 & 0.2005 & 0.105 & 0.095 & 0.228 & 0 & & 0 & 0 & & 0 & & $|0.027|$ & & & & 0 & & & & $\mid 0.0022$ & 0.007 & \\
\hline & 50.0359 & \begin{tabular}{|l|l|} 
& 0.1433 \\
\end{tabular} & 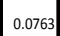 & \begin{tabular}{|l|l|} 
& 0.116 \\
\end{tabular} & 0.03 & \begin{tabular}{|l|l|} 
& 0.048 \\
\end{tabular} & 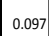 & & 。 & 0.0421 & 0.01 & $f_{1}$ & 0.081 & 0.005 & & 0.04 & 0.046 & & 0 & & $\mid 0.099$ & & & & & & & & & & \\
\hline & 0.0441 & 0.0305 & & \begin{tabular}{|l|l|} 
\\
\end{tabular} & 0.1278 & 0.133 & 0.044 & 0.0331 & 0.010 & 0.0319 & 0.006 & 0.023 & 0.017 & & & & 0.003 & & \begin{tabular}{|l|l|} 
& 0.0127 \\
\end{tabular} & 0.1101 & 0.122 & 0.081 & & & & & & & & & \\
\hline & & & 6E- 05 & & & 0.06 & 0.152 & & 0.01 & & & & & & & & 0.003 & & \begin{tabular}{|l|l|} 
& 0.0600 \\
\end{tabular} & 0.1593 & 0.104 & 0.033 & & & & & & & & & \\
\hline & & & & & & 0.007 & 0.162 & 0.0343 & & 0.0996 & & & & & & & 0.018 & & \begin{tabular}{|l|l|} 
\\
\end{tabular} & 0.0353 & 0.161 & 0.038 & 0.171 & & & & & & & & \\
\hline & & & & & & & \begin{tabular}{|l|l|} 
\\
\end{tabular} .015 & 0.0547 & & & & & & & & & & & 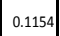 & 0.1269 & $|0.137|$ & \begin{tabular}{|l|l|} 
\\
\end{tabular} & $\mid 0.177$ & & & & & & & & 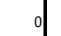 \\
\hline 10 & & & & & & & & 0.0047 & & & & & & & & & & & & 0.0361 & 0.091 & $|0.315|$ & 0.126 & $\mid 0.003$ & & & & & & & \\
\hline 11 & 0 & & & $\mid 0.311$ & 2.0877 & 0.055 & & & & & & & & & & & & & 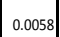 & 0.0431 & 0.069 & \begin{tabular}{|l|l|} 
\\
\end{tabular} & $0.065 \mid$ & \begin{tabular}{|l|l|} 
& 0.076
\end{tabular} & 0.03004 & & & & & & \\
\hline 12 & 0 & & & \begin{tabular}{|l|l|} 
\\
\end{tabular} & 2.701 & & & 0 & & & & & & & & & & & & 0.0574 & 0.201 & 0.097 & 0.041 & $\mid 0.035$ & & & & & & & 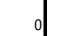 \\
\hline 13 & 0 & & & \begin{tabular}{|l|l|} 
\\
\end{tabular} & 4.123 & 0.036 & & 0 & 0 & & & & 0.211 & & & & 0 & 0.06 & 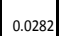 & 0.051 & 0.186 & 0.022 & & & & & & & & & \\
\hline 14 & 0 & & & \begin{tabular}{|l|l|} 
\\
\end{tabular} & 2.1218 & \begin{tabular}{|l|} 
\\
\end{tabular} & 5.14 & 0.88 & 0.199 & & & & 0.273 & 0.075 & & & & & & & 0 & & & 0.008 & 0.07049 & & & & & & \\
\hline 15 & 0 & & & & 0.4127 & \begin{tabular}{|l|} 
\\
\end{tabular} & 0.672 & 0.1839 & 0 & 0 & & & 0.071 & 0.133 & & & 0 & 0.01 & 0.0191 & 0 & 0 & & 0.013 & \begin{tabular}{|l|l|} 
\\
\end{tabular} .014 & 0.06749 & 0.03525 & \begin{tabular}{|l|l|} 
& 0.049
\end{tabular} & & & & \\
\hline 16 & 0 & & & & 0.9614 & 0.462 & 0.199 & 0.8855 & 0.08 & & 0 & & 0.08 & $\begin{array}{l}0.52 \\
\end{array}$ & & 0.562 & 0.931 & 0.066 & \begin{tabular}{|l|}
0.1637 \\
\end{tabular} & 0.7914 & 0.044 & & 0.057 & 0.21 & 0.06595 & 0.03377 & 0.00893 & 0 & & & \\
\hline 17 & & & & & & & & 0.0082 & 0 & & 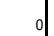 & & 0.04 & 0.099 & & 1.665 & 1.778 & 1.214 & \begin{tabular}{|l|l|} 
\\
\end{tabular} & 3.3223 & 0.034 & & 0.006 & $\mid 0.091$ & & 0.00123 & & 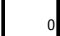 & & & 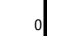 \\
\hline 18 & 0 & & & & & & & 0 & & & & & 0.015 & 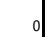 & & 1.114 & 0.736 & 0.451 & \begin{tabular}{|l|l|} 
& 1.4886 \\
\end{tabular} & 0.5188 & 0.008 & & 0.015 & \begin{tabular}{|l|l|} 
\\
\end{tabular} & 0.00948 & & & & & & 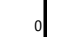 \\
\hline 19 & & & & & & & & & & & 0.044 & 0.638 & & & 1.566 & 0.689 & 0.121 & 0.428 & \begin{tabular}{|l|l|} 
\\
\end{tabular} & 0.1681 & 0.012 & & 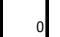 & & & & 0.08736 & \begin{tabular}{|l|l} 
\\
\end{tabular} & & & 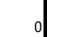 \\
\hline 20 & & & & & & & & 0.0887 & & & 0.746 & 0.593 & 0.04 & 0.874 & 2.015 & 0.707 & 0.541 & & \begin{tabular}{|l|l|} 
& 0.7212 \\
\end{tabular} & 0.8405 & & & 0.019 & & 0 & & 0.03761 & 0 & & & 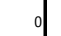 \\
\hline 21 & & & & & & 0.055 & \begin{tabular}{|l|}
1.897 \\
\end{tabular} & 2.3857 & 0.786 & & & & & 3.135 & 5.25 & 4.503 & 2.245 & 0.013 & 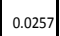 & 0 & & & & 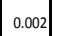 & 0.0613 & 0.07551 & 0.08554 & 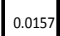 & & & \\
\hline 22 & 0 & & & & & & & & 0.417 & c & & ( & & 0.55 & 8.637 & 2.006 & 1.007 & d & 0 & & & & & & 0.11114 & 0.06815 & 0.00688 & 0.0032 & & 0.037 & 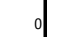 \\
\hline 23 & & & & & & & 0.17 & $\mid 0.1841$ & 0.17 & & 0.161 & & & & 3.516 & 1.743 & 0.382 & & 0 & & 0 & & 0.033 & 0.023 & 0.08598 & 0.10252 & & & 0.008 & 0.134 & 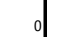 \\
\hline 24 & & & & & & & 0.182 & 0.0662 & 0.253 & & & 0.056 & & & & 0.041 & 0.061 & & & 0.0664 & 0.074 & 0.003 & 0.093 & 0.115 & 0.07352 & 0.06381 & 0.00934 & 0.0355 & 0.0372 & 0.061 & 0.041 \\
\hline
\end{tabular}


Table-3: Cluster-wise Magnitude of Velocity Corresponding to fig-7

\begin{tabular}{|c|c|c|c|c|c|c|c|c|c|c|c|c|c|c|c|c|c|c|c|c|c|c|c|c|c|c|c|c|c|c|c|c|}
\hline & & 2 & & & 5 & 6 & 7 & & 9 & 10 & 11 & 12 & 13 & 14 & 15 & 16 & 17 & 18 & 19 & 20 & 21 & 22 & 23 & 24 & 25 & 26 & 27 & 28 & 29 & 30 & 31 & \\
\hline & 0.008 & 5 & 0.031 & 0.07 & 0 & & & & & 0 & 0 & & & 0 & 0.02 & 0.02 & 0.01 & 0 & & & & & & & 0 & & & 0.01 & 0.57 & 0.48 & 0.07 & 0.03 \\
\hline & 0 & 0 & & & 0 & & 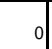 & & 0 & 0 & 0 & & & 0 & 0 & 0.26 & 0.07 & & & & & & & & 0 & & 0.1 & 0.14 & 3.04 & 0.39 & 0.33 & \\
\hline & 0.013 & & $\mid 0.009$ & & 0 & & 0 & & 0 & 0 & 0 & & 0 & 0 & 0.02 & 0.27 & 0.26 & 0.01 & & & 0 & 0 & 0 & & 0 & 0 & 0.22 & 0.04 & 0.98 & 0.14 & 0.21 & 0.0 \\
\hline & 0 & 0 & 0 & 0 & 0 & & 0 & & 0 & 0 & 0 & 0.05 & 0 & 0 & 0.05 & 0.08 & 0.08 & 158 & 2.339 & & 0 & & 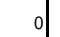 & & 0 & & & 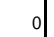 & & & 0.31 & 0.0 \\
\hline & 0 & 0 & 0 & 0 & 0 & & 0 & & 0 & 0 & 0 & 0.01 & 0 & 0 & 0.01 & 0.12 & 0.05 & 4.01 & 8 & & & & & & 0 & & & & & & 0.13 & \\
\hline & 0 & 0 & 0 & 0 & 0 & 0 & 0 & 0 & 0 & 0 & 0 & 0 & 0 & 0 & 0 & 0 & 0 & 5.24 & 1885 & & 0 & & & & 0 & & & & & & & \\
\hline & 0 & 0 & 0 & 0 & 0 & & 0 & \begin{tabular}{|l|} 
\\
\end{tabular} & 159 & 0 & 0.03 & 0 & 0 & \begin{tabular}{|l|}
0.12 \\
\end{tabular} & 5.48 & 3.48 & 156 & 111 & 69 & & . & & & & o & & & & & & & \\
\hline & 0 & 0 & 0 & 0 & 0 & 0 & 0.16 & \begin{tabular}{|l|} 
\\
\end{tabular} & 1.35 & 0 & 0 & ( & 0 & 0.32 & 4.52 & 18 & 0.62 & 2.37 & 3.544 & 106 & & & & & 0.99 & 2.61 & & & & & & \\
\hline & 0 & 0 & 0 & 0 & 0.03 & 0.22 & 0.46 & 2.03 & 2 & 0 & 0 & 0.47 & 103 & 0.31 & 5.56 & 161 & 0.16 & 3.84 & 0.508 & 0.5 & & & & & & 0.68 & 0.49 & & & & & \\
\hline $10 \mid$ & 0 & 0 & 0 & 0 & 3.08 & 8 & 0.84 & 136 & 3.42 & 122 & 0 & 0.46 & 3.32 & 0.61 & 5.36 & 0.27 & 0 & 0 & 3.258 & 2.72 & & 0.33 & 104 & & 0.87 & 1090 & 0.07 & & & & & \\
\hline 11 & 0 & 0 & & & 2.85 & ( & 0 & 2.22 & 3.85 & 0.47 & 0 & 0 & 0.79 & \begin{tabular}{|l|} 
\\
\end{tabular} & 0.41 & 0.27 & 0 & 0 & 1367 & 0.22 & 0.6 & 7.19 & 101 & & 2.87 & 15 & & & & & & \\
\hline 12 & 0 & & $\mid 0.308$ & 0.53 & 0.66 & 2. & 0 & \begin{tabular}{|l|l|} 
\\
\end{tabular} & 3.97 & 114 & 0 & 0.55 & 2.44 & 0 & 0 & 0.81 & 131 & 0.38 & 2.192 & 4.2 & 3.73 & \begin{tabular}{|l|}
10.4 \\
\end{tabular} & 7.93 & 153 & 0.36 & 0.16 & & & & & & \\
\hline 13 & 0 & 0 & 0.3 & 0.54 & 0.8 & 3.27 & 2.87 & \begin{tabular}{|l|}
3.38 \\
\end{tabular} & 5.81 & 0.94 & 106 & 0.81 & 0 & 0 & 0 & 0.07 & 0.15 & 122 & 8.058 & 4.43 & 119 & 3.05 & 0.36 & & 0 & & & & & & & \\
\hline 14 & 0 & 0 & & 0.1 & 181 & 0.9 & 0 & 1900 & 9.55 & 153 & 3.33 & 102 & 0.13 & 0 & 0 & 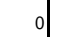 & 0 & 0.97 & 9.364 & 8.38 & 6.03 & 127 & 0.83 & & & 0.01 & & & & & & \\
\hline 15 & 0 & 0 & 0 & 0.01 & 0.05 & 0 & 0 & \begin{tabular}{|l|l|} 
& \\
\end{tabular} & 3.36 & 0.27 & 164 & 136 & 0.72 & \begin{tabular}{|l|}
0.04 \\
\end{tabular} & 0 & 0 & 0 & 0.04 & 2.678 & 5.33 & 5.27 & 8.42 & & & c & 0.03 & & & & & & \\
\hline 16 & 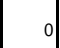 & 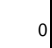 & 0 & l & 0 & 0 & 0 & & 2.82 & 125 & 0.91 & 0.89 & 0.14 & 0.06 & 0 & 0.01 & 0 & 0 & & $\mid 176$ & 2.73 & 2.15 & 0.33 & & 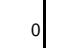 & & 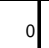 & & & & & \\
\hline 17 & 0 & 0 & 0 & 0 & 0 & 0 & 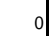 & & 0 & 0 & 0.82 & 4.54 & 0 & 0 & 0 & 0 & 0 & 158 & & 113 & 169 & 0.31 & 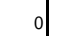 & & 0 & & & & & & & \\
\hline 18 & 0 & 0 & 0 & 0 & 0 & 0 & 0 & 0 & 0.01 & 0 & 156 & 153 & 18 & 0 & 0 & 0 & 0 & 0 & 1871 & 5.54 & 6.73 & 0 & o & & 0 & & & & & & & \\
\hline 19 & 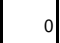 & 0 & 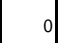 & d & 1 & 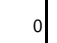 & 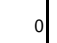 & & 0 & 0 & 0 & 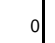 & 4 & 0 & 0 & 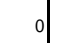 & 0 & 0 & & 7.15 & 6.09 & & 1 & & 0 & & 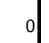 & & & & & \\
\hline 20 & 0 & 0 & 0 & 0 & 0 & 0 & 0 & 0 & 0 & 0 & 0 & 0 & 0 & 0 & 0 & 2.88 & 3.62 & 178 & 0.426 & 6.34 & 3.88 & 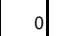 & 이 & & 0 & 0 & & 0 & & & & \\
\hline 21 & 0 & 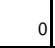 & & . & 0 & . & 0 & & 0 & 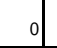 & 0 & 0.01 & 0.0 & 0 & 0 & 0.39 & 0.79 & 0 & 0.836 & 5.76 & 3.94 & 2.32 & 7 & & $5.4=$ & 2.98 & & & & & & \\
\hline 22 & 0 & 0 & 0 & 0 & 0 & 0 & 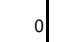 & & 0 & 0 & 0 & 0 & 0.02 & 0 & 0 & 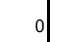 & of & 0.57 & 1132 & 123 & 0.3 & 2.57 & 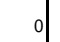 & & 3.98 & 2.89 & & 0 & & 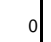 & & \\
\hline 23 & 0 & 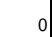 & 0 & 0 & 0 & 0 & 0 & & 0 & 0 & 0 & 0 & 0 & 0 & 0 & 0 & 0 & 0 & I & 0 & 3.98 & 0.44 & 0 & 0.46 & 3.22 & 4.92 & & 0.06 & & & & \\
\hline 2 & 0 & 0 & & & 0 & & 0 & & 0 & 0 & 0 & & & 0 & 0.04 & 0.01 & 0 & 0 & & & & 5.06 & & 4.66 & 5.53 & 163 & & 0.03 & & & & \\
\hline
\end{tabular}

\section{Conclusions}

People usually instinctively escape from a place when abnormal or dangerous events occur, we consider crowd escape behaviour as an indicator of an abnormal event. We proposed a method to detect anomalous crowd behaviours and its initial location.

The proposed method can detect and localize the abnormal behaviours happen. We conducted the experiments on the UMN dataset. We have made a comparative analysis with other methods and found that our method is more efficient to detect the abnormal events in crowed and its location.

Finally, we can detect and localize if any anomalous crowd behaviours present in the scene based on the energy of the optical flow.

\section{REFERENCES}

[1] Bruce D. Lucas "Generalized Image Matching by the Method of Differences”, Carnegie Mellon University, year 1984.

[2] J.L. Barron, D.J. Fleet, S.S. Beauchemin, T.A. Burkitt.” Performance of Optical Flow Techniques”, Computer Vision and Pattern Recognition, IEEE 1992.

[3] S. S. Beauchemin and J.L. Barron ,” The Computation of Optical Flow”, ACM Computing Survey (CSUR), volume 27,issue 3, pp. 433-466,1995.

[4] Hiroki Hayashi, Mineichi Kudo, Jun Toyama, and Masaru Shimbo, "Estimation of Velocity Vectors from a Video Stream Using Discontinuity of Optical Flow", Third International Conference on Knowledge-Based Intelligent Information Engineering Systems, Adelaide, Australia 3 1” Aug- Iw Sept 1999.

[5] N. M. Oliver, B. Rosario, and A. P. Pentland, “A bayesian computer vision system for modeling human interactions," IEEE TPAMI, vol. 22, no. 8, pp. 831-843, 2000.

[6] C. Stauffer and W. E. L. Grimson, "Learning patterns of activity using real-time tracking,” TPAMI, vol. 22, no. 8, pp. 747-757, 2000.

[7] D. Helbing, I. Farkas, and T. Vicsek, "Simulating dynamical features of escape panic ," Nature , vol. 407, no. 6803, pp. 487-490, doi: 10.1038/35035023, 2000.

[8] S. Kamijo, Y. Matsushita, K. Ikeuchi, and M. Sakauchi, “Traffic monitoring and accident detection at intersections,” IEEE Trans. ITS, vol. 1, no. 2, pp. 108-118, 2000.

[9] S.-F. Lin, J. -Y. Chen, and H.-X. Chao,“ Estimation of number of people in crowded scenes using perspective transformation,” IEEE Trans. Systems, Man, Cybern. A, vol. 31, no. 6, pp. 645-654, doi: 10.1109/3468.983420, 2001. 
[10] Mester R., "Some step towards a unified motion estimation procedure". In proceedings of the 45th IEEE Midwest symposium on circuits and systems, 2002.

[11] F. Niu and M. Abdel-Mottaleb, "View-invariant human activity recognition based on shape and motion features," in Proc. IEEE International Symposium on Multimedia Software Engineering, pp. 546-556, 2004.

[12] Ernest L. Andrade, Scott Blunsden and Robert B. Fisher, “Characterisation of Optical Flow Anomalies in Pedestrian Traffic", Imaging for Crime Detection and Prevention, 2005. ICDP DOI: 10.1049/ic: 20050073, 2005.

[13] P. Kumar, S. Ranganath, H. Weimin, and K. Sengupta, “Framework for real-time behaviour interpretation from traffic video," IEEE Trans. ITS, vol. 6, no. 1, pp. 43-53, 2005.

[14] Seyfried, A., Steffen, B., Klingsch, W., Boltes, M., "The Fundamental Diagram of Pedestrian Movement Revisited”, Journal of Statistical Mechanics-Theory and Experiment, p10002, 2005.

[15] E. L. Andradel, R. B. Fisher and Scott Blunsden, "Detection of Emergency Events in Crowded Scenes", IEEE conference on Crime and Security, 2006, London, pp. 528 - 533, IET, 2006.

[16] E. L. Andrade, S. Blunsden, and R. B. Fisher, "Hidden markov models for optical flow analysis in crowds," in Proc. ICPR, vol. 1, pp.460-463, 2006.

[17] Ernesto L. Andrade, Scott Blunsden and Robert B. Fisher,” Modelling Crowd Scenes for Event Detection”, Pattern Recognition, ICPR 2006, doi: 10.1109/ICPR.2006.806, pp. 175 - 178, Hong-Kong, 2006.

[18] Masaaki Shibata and Tomoki Yanagisawa “A Study of Optimal Angle of Colour Vector for Optical Flow Extraction”, SICE Annual Conference 2007, Kagawa University, Japan, Sept. 17-20, 2007.

[19] M. Hu, S. Ali, and M. Shah, "Detecting global motion patterns incomplex videos,” in Proc. ICPR, pp. 1-5, 2008.

[20] Tao Xiang and Shaogang Gong, "Incremental and adaptive abnormal behaviour detection,” CVIU, vol. 111, pp. 59-73, 2008.

[21] A. Chan and N. Vasconcelos, "Modeling, clustering, and segmenting video with mixtures of dynamic textures," IEEE Transactions on Pattern Analysis and Machine Intelligence, vol. 30, no. 5, pp. 909-926, doi: 10.1109/TPAMI.2007.70738, 2008.

[22] N. Ihaddadene and C. Djeraba, "Real-time crowd motion analysis," in Proc. ICPR, pp. 1-4, 2008.

[23] A. Adam, E. Rivlin, I. Shimshoni, and D. Reinitz, "Robust real-time unusual event detection using multiple fixed-location monitors," IEEE TPAMI, vol. 30, no. 3, pp. 555-560, 2008.

[24] Mehran R., Oyama, A., Shah, M., “Abnormal Crowd Behaviour Detection Using Social Force Model”, IEEE Conference on Computer Vision and Pattern Recognition, p. 935, 2009.

[25] Tian Cao, Xinyu Wu, Jinnian Guo, Shiqi Yu and Yangsheng Xu ”Abnormal Crowd Motion Analysis”, IEEE International Conference on Robotics and Biomimetics Guilin, China December 19 -23, 2009.

[26] Wei Wang, Peng Zhang, Runsheng Wang “Abnormal Video Sections Detection Based on Inter-Frames Information”, IEEE Third International Conference on Multimedia and Ubiquitous Engineering, 2009.

[27] Ali WALI, Adel M. ALIMI," Event Detection from video surveillance data based on Optical Flow Histogram and High-level feature extraction”, DOI 10.1109/DEXA.2009.81, IEEE, 2009

[28] Sukeshini N.Tamgade1, Vibha R.Bora2, "Motion vector estimation of video image by Pyramidal implementation of Lucas Kanade Optical flow”, IEEE, 2009’

[29] Shu Wang, Zhenjiang Miao, “Anomaly Detection in Crowd Scene”, ICSP2010 Proceedings, IEEE, 2010.

[30] J. C. S. J. Junior, S. R. Musse, and C. R. Jung, “Crowd analysis using computer vision techniques: A survey,” IEEE Signal Process. Lett. vol. 27, no. 5, pp. 66-77, doi: 10.1109/MSP.2010.937394, Sep. 2010.

[31] Abhijit Kundu, C. V. Jawahar and K Madhava Krishna "Real-time Moving Object Detection from a Freely Moving Monocular Camera”, IEEE, 2010.

[32] Christoph Hermes, Julian Einhaus, Markus Hahn, Christian W"ohler and Franz Kummert, "Vehicle Tracking and Motion Prediction in Complex Urban Scenarios", IEEE, 2010.

[33] C. Rao and M. Shah, "View-invariance in action recognition”, in Proc. IEEE CVPR, vol. 2, pp. 316-322, 2010.

[34] “Umn dataset, available from http://mha.cs.umn.edu/movies/crowdactivity-all.avi,” 2010.

[35] Lili Cui, Kehuang Li, Jiapin Chen, Zhenbo Li, “Abnormal Event Detection in Traffic Video Surveillance Based on Local Features”, IEEE, 2011.

[36] Su Yang, Wenbin Zhou,” Anomaly Detection on Collective Moving Patterns”, IEEE International Conference on Privacy, Security, Risk, and Trust, and IEEE International Conference on Social Computing, IEEE, 2011.

[37] X. Liu, L. Lin, S. Yan, H. Jin, and W. Tao, "Integrating spatiotemporal context with multi view representation for object recognition in visual surveillance," IEEE Transactions on Circuits and Systems for Video Technology, vol. 21, no. 4, pp. 393-407, doi: 10.1109/TCSVT.2010.2087570, 2011.

[38] Chen, D. Y., Huang, P. C., "Motion-based Unusual Event Detection in Human Crowds", Journal of Visual Communication and Image Representation 22, p. 1782011.

[39] Mahfuzul Haque and Manzur Murshed, "Abnormal Event Detection in Unseen Scenarios”, 2012 IEEE International Conference on Multimedia and Expo Workshops DOI 10.1109/ICMEW.2012.72, IEEE, 2012.

[40] Hajananth Nallaivarothayan, David Ryan, Simon Denman, Sridha Sridharan and Clinton Fookes, “Anomalous Event Detection using a Semi-Two Dimensional Hidden Markov Model”, IEEE, 2012.

[41] Tian Wang, Hichem Snoussi,” Histograms of Optical Flow Orientation for Visual Abnormal Events Detection”, 2012 IEEE Ninth International Conference on Advanced Video and Signal-Based Surveillance, DOI 10.1109/AVSS.2012.39, IEEE, 2012.

[42] Hiral Raveshia and Viral Boris agar, ” Motion Estimation Using Optical Flow Concepts”. International journal of computer technology and applications, vol 3(2), pp. 696-700, 2012.

[43] Nusrat Sharmin and Remus Brad,” Optimal Filter Estimation for Lucas-Kanade Optical Flow”, Sensors 2012, 12, 12694-12709; doi: 10.3390/s120912694, 2012.

[44] Popoola, O. P., Wang, K., "Video-Based abnormal human behaviour recognition—a review," IEEE Trans. Syst. Man Cybern Part C vol.42, pp. 865-878, doi: 10.1109/ TSMCC.2011.2178594, 2012.

[45] Hajananth Nallaivarothayan, David Ryan, Simon Denman, Sridha Sridharan and Clinton Fookes, "An Evaluation of Different Features and Learning Models for Anomalous Event Detection” Digital Image Computing: Techniques and Applications (DICTA), IEEE, 2013.

[46] Hiba H. Alqaysi and Sreela Sasi, "Detection of Abnormal behaviour in Dynamic Crowded Gatherings “, Pages: 1 - 6, DOI: 10.1109/ AIPR.2013.6749309, IEEE, 2013.

[47] Weiguo Song, Wei Lv, Zhiming Fang, "Experiment and modeling of microscopic movement characteristic of pedestrians”, Procedia Engineering 62, pp 56 - 70, 2013

[48] Huy-Hung Nguyen, Phuong-Tai Tran-Huu, Ha Manh Tran, Synh Viet-Uyen Ha, "Improved Optical Flow Estimation In Traffic Monitoring System”, PP. 165 - 169, DOI: 10.1109/WICT.2013.7113129, IEEE, 2013.

[49] T. Malavika, M. Poornima," Moving object detection and velocity estimation using MATLAB”, International Journal of Engineering Research \& Technology (IJERT) Vol. 2 Issue 1, January- 2013. 
[50] Dhara Patel, Saurabh Upadhyay,” Optical Flow Measurement using Lucas kanade Method”, International Journal of Computer Applications (0975 - 8887) Volume 61- No.10, January 2013

[51] Soheil Bahrampour, Asok Ray, Soumalya Sarkar, Thyagaraju Damarla, Nasser M. Nasrabadi, "Performance comparison of feature extraction algorithms for target detection and classification q", Pattern Recognition Letters 34 , PP. 2126-2134, 2013.

[52] Jorge Henrique Busatto Casagrande, Marcelo Ricardo Stemmer,” Abnormal Motion Analysis for Tracking-Based Approaches Using Region-Based Method with Mobile Grid”, Journal of Image and Graphics, Volume 2, No.1, doi: 10.12720/joig.2.1.22-27June 2014.

[53] C. Rajanayaki, K.G. Srinivasagan, S. Kalaiselvi, "An Efficient Method for Crowd Event Recognition based on Motion patterns", International Conference on Recent Trends in Information Technology, IEEE, 2014.

[54] Chunyu Chen, Yu Shao”' Anomalous Crowd Behaviour Detection and Localization in Video Surveillance”, IEEE, 2014.

[55] Tian Wang, and Hichem Snoussi, "Detection of Abnormal Visual Events via Global Optical Flow Orientation Histogram", IEEE TRANSACTIONS ON INFORMATION FORENSICS AND SECURITY, VOL. 9, NO. 6, JUNE 2014.

[56] Aravinda S. Rao, Jayavardhana Gubbi, Slaven Marusic, and Marimuthu Palaniswami, "Crowd Event Detection on Optical Flow Manifolds”, IEEE TRANSACTIONS ON CYBERNETICS 1 Digital Object Identifier 10.1109/TCYB.2015.2451136, PP. 2168-2267, IEEE, 2015.

[57] A. F. M. Saifuddin Saif, Anton Satria Prabuwono and Zainal Rasyid Mahayuddin, "Moment Feature Based Fast Feature Extraction Algorithm for Moving Object Detection Using Aerial Images”, DOI: 10.1371/journal. Pone. 0126212, June 1, 2015

[58] Weixin Li, Vijay Mahadevan, and Nuno Vasconcelos,” Anomaly Detection and Localization in Crowded Scenes” IEEE Transaction Pattern Analyses and Machine Intelligence, Vol. 36, N0. 1, January 2014. 